\title{
Iterative Learning of Answer Set Programs from Context Dependent Examples
}

\author{
Mark Law, Alessandra Russo*, Krysia Broda \\ Department of Computing, Imperial College London, SW7 $2 A Z$ \\ (e-mail: \{mark.law09, a.russo, k.broda\}@imperial.ac.uk ) \\ submitted 1 January 2003; revised 1 January 2003; accepted 1 January 2003
}

\begin{abstract}
In recent years, several frameworks and systems have been proposed that extend Inductive Logic Programming (ILP) to the Answer Set Programming (ASP) paradigm. In ILP, examples must all be explained by a hypothesis together with a given background knowledge. In existing systems, the background knowledge is the same for all examples; however, examples may be context-dependent. This means that some examples should be explained in the context of some information, whereas others should be explained in different contexts. In this paper, we capture this notion and present a context-dependent extension of the Learning from Ordered Answer Sets framework. In this extension, contexts can be used to further structure the background knowledge. We then propose a new iterative algorithm, ILASP2i, which exploits this feature to scale up the existing ILASP2 system to learning tasks with large numbers of examples. We demonstrate the gain in scalability by applying both algorithms to various learning tasks. Our results show that, compared to ILASP2, the newly proposed ILASP2i system can be two orders of magnitude faster and use two orders of magnitude less memory, whilst preserving the same average accuracy. This paper is under consideration for acceptance in TPLP.
\end{abstract}

KEYWORDS: Non-monotonic Inductive Logic Programming, Answer Set Programming, Iterative Learning

\section{Introduction}

Inductive Logic Programming (Muggleton 1991) (ILP) addresses the task of learning a logic program, called a hypothesis, that explains a set of examples using some background knowledge. Although ILP has traditionally addressed learning (monotonic) definite logic programs, recently, several new systems have been proposed for learning under the (non-monotonic) answer set semantics (e.g. (Ray 2009), (Corapi et al. 2012), (Athakravi et al. 2014), (Law et al. 2014) and (Law et al. 2015a)). Among these, ILASP2 (Law et al. 2015a) extended ILP to learning from ordered answer sets $\left(I L P_{L O A S}\right)$, a computational task that learns answer set programs containing normal rules, choice rules and both hard and weak constraints.

Common to all ILP systems is the underlying assumption that hypotheses should

* This research is partially funded by the EPSRC project EP/K033522/1 "Privacy Dynamics". 
cover the examples with respect to one fixed given background knowledge. But, in practice, some examples may be context-dependent - different examples may need to be covered using different background knowledges. For instance, within the problem domain of urban mobility, the task of learning journey preferences of people in a city may require a general background knowledge that describes the different modes of transport available to a user (walk, drive, etc.), and examples of which modes of transport users choose for particular journeys. In this case, the context of an example would be the attributes (e.g. the distance) of the journey. It is infeasible to assume that every possible journey could be encoded in the background knowledge - attributes, such as journey distances, may take too many possible values. But, encoding the attributes of observed journeys as contexts of the observations restricts the computation to those attribute values that are in the contexts.

In this paper, we present a generalisation of $I L P_{L O A S}$, called context-dependent learning from ordered answer sets $\left(I L P_{L O A S}^{c o n t e x t}\right)$, which uses context-dependent examples. We show that any $I L P_{L O A S}^{\text {context }}$ task can be translated into an $I L P_{L O A S}$ task, and can therefore be solved by ILASP2. Furthermore, to improve the scalability of ILASP2, we present a new iterative reformulation of this learning algorithm, called ILASP2i. This iterative approach differs from existing non-monotonic learning systems, which tend to be batch learners, meaning that they consider all examples at once. Non-monotonic systems cannot use a traditional cover loop (e.g., (Muggleton 1995)), as examples that were covered in previous iterations are not guaranteed to be covered in later iterations. However, ILASP2i iteratively computes a hypothesis by constructing a set of examples that are relevant to the search, without the need to consider all examples at once. Relevant examples are essentially counterexamples for the hypotheses found in previous iterations. This approach is a middle ground between batch learning and the cover loop: it avoids using the whole set of examples, but works in the non-monotonic case, as the relevant examples persist through the iterations. We show that ILASP2i performs significantly better than ILASP2 in solving learning from ordered answer set tasks with large numbers of examples, and better still when learning with context-dependent examples, as in each iteration it only considers the contexts of relevant examples, rather than the full set.

To demonstrate the increase in scalability we compare ILASP2i to ILASP2 on a variety of tasks from different problem domains. The results show that ILASP2i is up to 2 orders of magnitude faster and uses up to 2 orders of magnitude less memory than ILASP2. We have also applied both algorithms to the real-world problem domain of urban mobility, and explored in greater depth the task of learning a user's journey preferences from pairwise examples of which journeys are preferred to others. As we learn ASP, these user preferences can very naturally be represented as weak constraints, which give an ordering over the journeys. Our results show that ILASP2i achieves an accuracy of at least $85 \%$ with around 40 examples. We also show that, by further extending $I L P_{L O A S}^{c o n t e x t}$ with ordering examples that express equal preferences, in addition to strict ordering, the accuracy can increase to $93 \%$.

The rest of the paper is structured as follows. In Section 2 we review the relevant background. In Section 3 we present our new context-dependent learning from ordered answer set task, and in Section 4 we introduce our new ILASP2i algorithm. In 
Section 5 we compare ILASP2i to ILASP2 on a range of different learning tasks and give a detailed evaluation of the accuracy of ILASP2i and compare its scalability with ILASP2 in the context of the journey planning problem. Finally, we conclude the paper with a discussion of related and future work.

\section{Background}

Let $\mathrm{h}, \mathrm{h}_{1}, \ldots, \mathrm{h}_{\mathrm{k}}, \mathrm{b}_{1}, \ldots, \mathrm{b}_{\mathrm{n}}$ be atoms and $\mathrm{l}$ and $\mathrm{u}$ be integers. The ASP programs we consider contain normal rules, of the form $h:-b_{1}, \ldots, b_{m}$, not $b_{m+1}, \ldots$, not $b_{n}$; constraints, which are rules of the form :- $b_{1}, \ldots, b_{m}$, not $b_{m+1}, \ldots$, not $b_{n}$; and choice rules, of the form $l\left\{h_{1}, \ldots, h_{k}\right\} u:-b_{1}, \ldots, b_{m}$, not $b_{m+1}, \ldots$, not $b_{n}$. We refer to the part of the rule before the ":-" as the head, and the part after the ":-" as the body. The meaning of a rule is that if the body is true, then the head must be true. The empty head of a constraint means false, and constraints are used to rule out answer sets. The head of a choice rule is true if between $I$ and $u$ atoms from $\mathrm{h}_{1}, \ldots, \mathrm{h}_{\mathrm{k}}$ are true. The solutions of an ASP program $P$ form a subset of the Herbrand models of $P$, called the answer sets of $P$ and denoted as $A S(P)$.

ASP also allows optimisation over the answer sets according to weak constraints, which are rules of the form $: \sim b_{1}, \ldots, b_{m}$, not $b_{m+1}, \ldots$, not $b_{n} \cdot\left[w @ p, t_{1}, \ldots, t_{k}\right]$ where $b_{1}, \ldots, b_{n}$ are atoms called (collectively) the body of the rule, and $w, p, t_{1} \ldots t_{k}$ are all terms with $w$ called the weight and $\mathrm{p}$ the priority level. We will refer to $\left[\mathrm{w} @ \mathrm{p}, \mathrm{t}_{1}, \ldots, \mathrm{t}_{\mathrm{k}}\right]$ as the tail of the weak constraint. A ground instance of a weak constraint $W$ is obtained by replacing all variables in $W$ (including those in the tail of $W$ ) with ground terms. In this paper, it is assumed that all weights and levels of all ground instances of weak constraints are integers.

Given a program $P$ and an interpretation $I$ we can construct the set of tuples $\left(\mathrm{w}, \mathrm{p}, \mathrm{t}_{1}, \ldots, \mathrm{t}_{\mathrm{k}}\right)$ such that there is a ground instance of a weak constraint in $P$ whose body is satisfied by $I$ and whose (ground) tail is $\left[\mathrm{w} @ \mathrm{p}, \mathrm{t}_{1}, \ldots, \mathrm{t}_{\mathrm{k}}\right]$. At each level $\mathrm{p}$ the score of $I$ is the sum of the weights of tuples with level p. An interpretation $I_{1}$ dominates another interpretation $I_{2}$ if there is a level $\mathrm{p}$ for which $I_{1}$ has a lower score than $I_{2}$, and no level higher than $\mathrm{p}$ for which the scores of $I_{1}$ and $I_{2}$ are unequal. We write $I_{1} \prec_{P} I_{2}$ to denote that given the weak constraints in $P, I_{1}$ dominates $I_{2}$.

\section{Example 1}

Consider the set $W S=\left\{\begin{array}{l}: \sim \operatorname{mode}(\mathrm{L}, \text { walk }), \operatorname{crime} \_\operatorname{rating}(\mathrm{L}, \mathrm{R}), \mathrm{R}>3 .[1 @ 3, \mathrm{~L}, \mathrm{R}] \\ : \sim \operatorname{mode}(\mathrm{L}, \text { bus }) .[1 @ 2, \mathrm{~L}] \\ : \sim \operatorname{mode}(\mathrm{L}, \text { walk }), \operatorname{distance}(\mathrm{L}, \mathrm{D}) .[\mathrm{D} @ 1, \mathrm{~L}, \mathrm{D}]\end{array}\right.$

The first weak constraint in $W S$, at priority 3 , means "minimise the number of legs in our journey in which we have to walk through an area with a crime rating higher than 3". As this has the highest priority, answer sets are evaluated over this weak constraint first. The remaining weak constraints are considered only for those answer sets that have an equal number of legs where we have to walk through an area with such a crime rating. The second weak constraint means "minimise the number of buses we have to take" (at priority 2). Finally, the last weak constraint means "minimise the distance walked". Note that this is the case because for each 
leg where we have to walk, we pay the penalty of the distance of that leg (so the total penalty at level 1 is the sum of the distances of the walking legs).

We now briefly summarise the key properties of Learning from Ordered Answer Sets and ILASP2, which we extend in this paper to Context-dependent Learning from Ordered Answer Sets and ILASP2i. It makes use of two types of examples: partial interpretations and ordering examples. A partial interpretation $e$ is a pair of sets of atoms $\left\langle e^{i n c}, e^{e x c}\right\rangle$. An answer set $A$ extends $e$ if $e^{i n c} \subseteq A$ and $e^{e x c} \cap A=\emptyset$. An ordering example is a pair of partial interpretations. A program $P$ bravely (resp. cautiously) respects an ordering example $\left\langle e_{1}, e_{2}\right\rangle$ if for at least one (resp. every) pair of answer sets $\left\langle A_{1}, A_{2}\right\rangle$ that extend $e_{1}$ and $e_{2}$, it is the case that $A_{1} \prec_{P} A_{2}$.

\section{Definition 1}

(Law et al. 2015a) A Learning from Ordered Answer Sets $\left(I L P_{L O A S}\right)$ task $T$ is a tuple $\left\langle B, S_{M}, E\right\rangle$ where $B$ is an ASP program, called the background knowledge, $S_{M}$ is the set of rules allowed in hypotheses (the hypothesis space) and $E$ is a tuple $\left\langle E^{+}, E^{-}, O^{b}, O^{c}\right\rangle . E^{+}$and $E^{-}$are finite sets of partial interpretations called, respectively, positive and negative examples. $O^{b}$ and $O^{c}$ are finite sets of ordering examples over $E^{+}$called, respectively, brave and cautious orderings. A hypothesis $H$ is an inductive solution of $T$ (written $H \in I L P_{L O A S}(T)$ ) iff: $H \subseteq S_{M} ; \forall e \in E^{+}$, $\exists A \in A S(B \cup H)$ st $A$ extends $e ; \forall e \in E^{-}, \nexists A \in A S(B \cup H)$ st $A$ extends $e$; $\forall o \in O^{b}, B \cup H$ bravely respects $o$; and, $\forall o \in O^{c}, B \cup H$ cautiously respects $o$.

In (Law et al. 2015a), we proposed a learning algorithm, called ILASP2, and proved that it is sound and complete with respect to ILPLOAS tasks. We use the notation $I L A S P 2\left(\left\langle B, S_{M}, E\right\rangle\right)$ to denote a function that uses ILASP2 to return an optimal (shortest in terms of number of literals) solution of the task $\left\langle B, S_{M}, E\right\rangle$. ILASP2 terminates for any task such that $B \cup S_{M}$ grounds finitely (or equivalently, $\forall H \subseteq S_{M}, B \cup H$ grounds finitely). We call any such task well defined.

\section{Context-dependent Learning from Ordered Answer Sets}

In this section, we present an extension to the $I L P_{L O A S}$ framework called Contextdependent Learning from Ordered Answer Sets (written $I L P_{L O A S}^{c o n t e x t}$ ). In this new learning framework, examples can be given with an extra background knowledge called the context of an example. The idea is that each context only applies to a particular example, giving more structure to the background knowledge.

\section{Definition 2}

A context-dependent partial interpretation (CDPI) is a pair $\langle e, C\rangle$, where $e$ is a partial interpretation and $C$ is an ASP program with no weak constraints, called a context. A context-dependent ordering example (CDOE) $o$ is a pair of CDPIs, $\left\langle\left\langle e_{1}, C_{1}\right\rangle,\left\langle e_{2}, C_{2}\right\rangle\right\rangle$. A program $P$ is said to bravely (resp. cautiously) respect $o$ if for at least one (resp. every) pair $\left\langle A_{1}, A_{2}\right\rangle$ such that $A_{1} \in A S\left(P \cup C_{1}\right), A_{2} \in A S\left(P \cup C_{2}\right)$, $A_{1}$ extends $e_{1}$ and $A_{2}$ extends $e_{2}$, it is the case that $A_{1} \prec_{P} A_{2}$. 
Example 2

Consider the programs $P=\{\operatorname{coin}(1 \ldots 2) .1\{\operatorname{val}(\mathrm{C}, \mathrm{h}), \operatorname{val}(\mathrm{C}, \mathrm{t})\} 1:-\operatorname{coin}(\mathrm{C})$.$\} ,$ $C_{1}=\{\operatorname{val}(1, \mathrm{v}):-\operatorname{val}(2, \mathrm{v}) \cdot\}$ and $C_{2}=\{:-\operatorname{val}(1, \mathrm{v}), \operatorname{val}(2, \mathrm{v}) \cdot\} \cdot A S\left(P \cup C_{1}\right)=$ $\{\{\operatorname{val}(1, \mathrm{~h}), \operatorname{val}(2, \mathrm{~h})\},\{\operatorname{val}(1, \mathrm{t}), \operatorname{val}(2, \mathrm{t})\}\}$ and $A S\left(P \cup C_{2}\right)=\{\{\operatorname{val}(1, \mathrm{~h})$, $\operatorname{val}(2, \mathrm{t})\},\{\operatorname{val}(1, \mathrm{t}), \operatorname{val}(2, \mathrm{~h})\}\}$. Also consider the CDOE $o=\left\langle\left\langle e_{1}, C_{1}\right\rangle,\left\langle e_{2}, C_{2}\right\rangle\right\rangle$, where $e_{1}=e_{2}=\langle\emptyset, \emptyset\rangle$, Let $W=\{: \sim \operatorname{val}(\mathrm{C}, \mathrm{t}) \cdot[1 @ 1, \mathrm{C}]\} . P \cup W$ bravely respects $o$ as $\{\operatorname{val}(1, \mathrm{~h}), \operatorname{val}(2, \mathrm{~h})\}$ is preferred to $\{\operatorname{val}(1, \mathrm{~h}), \operatorname{val}(2, \mathrm{t})\}$, but does not cautiously respect $o$ as $\{\operatorname{val}(1, \mathrm{t}), \operatorname{val}(2, \mathrm{t})\}$ is not preferred to $\{\operatorname{val}(1, \mathrm{~h}), \operatorname{val}(2, \mathrm{t})\}$.

Examples with empty contexts are equivalent to examples in $I L P_{L O A S}$. Note that contexts do not contain weak constraints. The operator $\prec_{P}$ defines the ordering over two answer sets based on the weak constraints in one program $P$. So, given a CDOE $\left\langle\left\langle e_{1}, C_{1}\right\rangle,\left\langle e_{2}, C_{2}\right\rangle\right\rangle$, in which $C_{1}$ and $C_{2}$ contain different weak constraints, it is not clear whether the ordering should be checked using the weak constraints in $P, P \cup C_{1}, P \cup C_{2}$ or $P \cup C_{1} \cup C_{2}$. We now present the $I L P_{L O A S}^{\text {context }}$ framework.

Definition 3

A Context-dependent Learning from Ordered Answer Sets (ILP $\left.P_{L O A S}^{\text {context }}\right)$ task is a tuple $T=\left\langle B, S_{M}, E\right\rangle$ where $B$ is an ASP program called the background knowledge, $S_{M}$ is the set of rules allowed in the hypotheses (the hypothesis space) and $E$ is a tuple $\left\langle E^{+}, E^{-}, O^{b}, O^{c}\right\rangle$ called the examples. $E^{+}$and $E^{-}$are finite sets of CDPIs called, respectively, positive and negative examples, and $O^{b}$ and $O^{c}$ are finite sets of CDOEs over $E^{+}$called, respectively, brave and cautious orderings. A hypothesis $H$ is an inductive solution of $T$ (written $H \in I L P_{L O A S}^{\text {context }}(T)$ ) if and only if:

1. $H \subseteq S_{M}$;

2. $\forall\langle e, C\rangle \in E^{+}, \exists A \in A S(B \cup C \cup H)$ st $A$ extends $e$;

3. $\forall\langle e, C\rangle \in E^{-}, \nexists A \in A S(B \cup C \cup H)$ st $A$ extends $e$;

4. $\forall o \in O^{b}, B \cup H$ bravely respects $o$; and finally,

5. $\forall o \in O^{c}, B \cup H$ cautiously respects $o$.

In this paper we will say a hypothesis covers an example iff it satisfies the appropriate condition in (2)-(5); e.g. a brave CDOE is covered iff it is bravely respected.

Example 3

In general, it is not the case that an $I L P_{L O A S}^{\text {context }}$ task can be translated into an $I L P_{L O A S}$ task simply by moving all the contexts into the background knowledge $\left(B \cup C_{1} \cup \ldots \cup C_{n}\right.$ where $C_{1}, \ldots, C_{n}$ are the contexts of the examples). Consider, for instance, the $I L P_{L O A S}^{\text {context }}$ task $\left\langle B, S_{M},\left\langle E^{+}, E^{-}, O^{b}, O^{c}\right\rangle\right\rangle$ defined as follows:

- $B=\emptyset \cdot E^{-}=\emptyset \cdot O^{b}=\emptyset \cdot O^{c}=\emptyset$

- $S_{M}=\{$ go_out:- raining. go_out:- not raining. $\}$

- $E^{+}=\{\langle\langle\{$go_out $\}, \emptyset\rangle, \emptyset\rangle,\langle\langle\emptyset,\{$ go_out $\}\rangle,\{$ raining. $\}\rangle\}$

This task has one solution: go_out:- not raining. But, if we were to add all the contexts to the background knowledge, we would get a background knowledge containing the single fact raining. So, there would be no way of explaining both examples, as every hypothesis would, in this case, lead to a single answer set (either \{raining, go_out\} or \{raining\}), and therefore cover only one of the examples. 
To capture, instead, the meaning of context-dependent examples accurately, we could augment the background knowledge with the choice rule $0\{$ raining 1 and define the $I L P_{L O A S}$ examples as the pairs $\langle\{$ go_out $\},\{$ raining $\}\rangle$ and $\langle\{$ raining $\}$, $\{$ go_out $\}\rangle$. In this way, answer sets of the inductive solution would exclude go_out when raining (i.e., in the context of raining), and include go_out otherwise, which is the correct meaning of the given context-dependent examples.

Definition 4 gives a general translation of $I L P_{L O A S}^{\text {context }}$ to $I L P_{L O A S}$, which enables the use of ILASP2 to solve $I L P \underset{L O A S}{\text { context }}$ tasks. The translation assumes that each example $e x$ has a unique (constant) identifier, $e_{i d}$, and that for any CDPI $e x=$ $\left\langle\left\langle e^{i n c}, e^{e x c}\right\rangle, C\right\rangle, c(e x)$ is the partial interpretation $\left\langle e^{i n c} \cup\left\{\operatorname{ctx}\left(\operatorname{ex}_{\mathrm{id}}\right)\right\}, e^{e x c}\right\rangle$, where ctx is a new predicate. Also, for any program $P$ and any atom a, $\operatorname{append}(P, a)$ is the program constructed by appending a to the body of every rule in $P$.

Definition 4

For any $I L P_{L O A S}^{\text {context }} \operatorname{task} T=\left\langle B_{1}, S_{M},\left\langle E_{1}^{+}, E_{1}^{-}, O_{1}^{b}, O_{1}^{c}\right\rangle\right\rangle, \mathcal{T}_{L O A S}(T)=\left\langle B_{2}, S_{M}\right.$, $\left.\left\langle E_{2}^{+}, E_{2}^{-}, O_{2}^{b}, O_{2}^{c}\right\rangle\right\rangle$, where the components of $\mathcal{T}_{L O A S}(T)$ are as follows:

- $B_{2}=B_{1} \cup\left\{\operatorname{append}\left(C, \operatorname{ctx}\left(\operatorname{ex}_{\mathrm{id}}\right)\right) \mid e x=\langle e, C\rangle \in E_{1}^{+} \cup E_{1}^{-}\right\}$

$\cup\left\{1\left\{\operatorname{ctx}\left(\mathrm{id}_{1}\right), \ldots, \operatorname{ctx}\left(\mathrm{id}_{\mathrm{n}}\right)\right\} 1 . \mid\left\{i d_{1}, \ldots, i d_{n}\right\}=\left\{e x_{i d} \mid e x \in E_{1}^{+} \cup E_{1}^{-}\right\}\right\}$

- $E_{2}^{+}=\left\{c(e x) \mid e x \in E_{1}^{+}\right\} ; E_{2}^{-}=\left\{c(e x) \mid e x \in E_{1}^{-}\right\}$

- $O_{2}^{b}=\left\{\left\langle c\left(e x_{1}\right), c\left(e x_{2}\right)\right\rangle \mid\left\langle e x_{1}, e x_{2}\right\rangle \in O_{1}^{b}\right\} ; O_{2}^{c}=\left\{\left\langle c\left(e x_{1}\right), c\left(e x_{2}\right)\right\rangle \mid\left\langle e x_{1}, e x_{2}\right\rangle \in O_{1}^{c}\right\}$

We say that an $I L P_{L O A S}^{\text {context }}$ task $T$ is well defined if and only if $\mathcal{T}_{L O A S}(T)$ is a well defined $I L P_{L O A S}$ task. Before proving that this translation is correct, it is useful to introduce a lemma (which is proven in Appendix A). Given a program $P$ and a set of contexts $C_{1}, \ldots, C_{n}$, Lemma 1 gives a way of combining the alternative contexts into the same program. Each rule of each context $C_{i}$, is appended with a new atom $\mathrm{a}_{\mathrm{i}}$, unique to $C_{i}$, and a choice rule stating that exactly one of the new $\mathrm{a}_{\mathrm{i}}$ atoms is true in each answer set. This means that the answer sets of $P \cup C_{i}$, for each $C_{i}$, are the answer sets of the combined program that contain $a_{i}$ (with the extra atom $a_{i}$ ).

\section{Lemma 1}

For any program $P$ (consisting of normal rules, choice rules and constraints) and any set of pairs $S=\left\{\left\langle C_{1}, \mathrm{a}_{1}\right\rangle, \ldots,\left\langle C_{n}, \mathrm{a}_{\mathrm{n}}\right\rangle\right\}$ such that none of the atoms $\mathrm{a}_{\mathrm{i}}$ appear in $P$ (or in any of the $C$ 's) and each $\mathrm{a}_{\mathrm{i}}$ atom is unique: $A S\left(P \cup\left\{1\left\{\mathrm{a}_{1}, \ldots, \mathrm{a}_{\mathrm{n}}\right\} 1.\right\} \cup\right.$ $\left.\left\{\operatorname{append}\left(C_{i}, \mathrm{a}_{\mathrm{i}}\right) \mid\left\langle C_{i}, \mathrm{a}_{\mathrm{i}}\right\rangle \in S\right\}\right)=\left\{A \cup\left\{\mathrm{a}_{\mathrm{i}}\right\} \mid A \in A S\left(P \cup C_{i}\right),\left\langle C_{i}, \mathrm{a}_{\mathrm{i}}\right\rangle \in S\right\}$

Theorem 1

For any $I L P_{L O A S}^{\text {context }}$ learning task $T, I L P_{L O A S}\left(\mathcal{T}_{L O A S}(T)\right)=\operatorname{ILP}_{L O A S}^{\text {context }}(T)$.

\section{Proof}

Let $T=\left\langle B_{1}, S_{M},\left\langle E_{1}^{+}, E_{1}^{-}, O_{1}^{b}, O_{1}^{c}\right\rangle\right\rangle$ and $\mathcal{T}_{L O A S}(T)=\left\langle B_{2}, S_{M},\left\langle E_{2}^{+}, E_{2}^{-}, O_{2}^{b}, O_{2}^{c}\right\rangle\right\rangle$. $H \in I L P_{L O A S}^{\text {context }}(T) \Leftrightarrow H \subseteq S_{M} ; \forall\langle e, C\rangle \in E_{1}^{+}, \exists A \in A S\left(B_{1} \cup C \cup H\right)$ st $A$ extends $e ; \forall\langle e, C\rangle \in E_{1}^{-}, \nexists A \in A S\left(B_{1} \cup C \cup H\right)$ st $A$ extends $e ; \forall o \in O_{1}^{b}, B_{1} \cup H$ bravely respects $o ; \forall o \in O_{1}^{c}, B_{1} \cup H$ cautiously respects $o$

$\Leftrightarrow H \subseteq S_{M} ; \forall e x \in E_{1}^{+}, \exists A \in A S\left(B_{2} \cup H\right)$ st $A$ extends $c(e x) ; \forall e x \in E_{1}^{-}, \nexists A \in$ 
$A S\left(B_{2} \cup H\right)$ st $A$ extends $c(e x) ; \forall\left\langle e x_{1}, e x_{2}\right\rangle \in O^{b}, B_{2} \cup H$ bravely respects $\left\langle c\left(e x_{1}\right)\right.$, $\left.c\left(e x_{2}\right)\right\rangle ; \forall\left\langle e x_{1}, e x_{2}\right\rangle \in O^{c}, B_{2} \cup H$ cautiously respects $\left\langle c\left(e x_{1}\right), c\left(e x_{2}\right)\right\rangle$ (by Lemma 1 ) $\Leftrightarrow H \subseteq S_{M} ; \forall e \in E_{2}^{+}, \exists A \in A S\left(B_{2} \cup H\right)$ st $A$ extends $e ; \forall e \in E_{2}^{-}, \nexists A \in A S\left(B_{2} \cup H\right)$ st $A$ extends $e ; \forall o \in O^{b}, B_{2} \cup H$ bravely respects $o ; \forall o \in O^{c}, B_{2} \cup H$ cautiously respects $O$

Theorem 1 shows that, by using an automatic $\mathcal{T}_{L O A S}$ translation, ILASP2 can be used to solve $I L P_{L O A S}^{\text {context }}$ tasks. Although this means that any $I L P_{L O A S}^{\text {context }}$ task can be translated to an $I L P_{L O A S}$ task, context-dependent examples are useful for two reasons: firstly, they simplify the representation of some learning tasks; and secondly, the added structure gives more information about which parts of the background knowledge apply to particular examples. In Section 4 we present a new algorithm that is able to take advantage of this extra information.

Theorem 2

The complexity of deciding whether an $I L P_{L O A S}^{\text {context }}$ task is satisfiable is $\Sigma_{2}^{P}$-complete.

Theorem 2 (proven in Appendix A implies that the complexity of deciding the satisfiability of an $I L P_{L O A S}^{\text {context }}$ task is the same as for an $I L P_{L O A S}$ task. Note that, similar to Theorem 2 in (Law et al. 2015a), this result is for propositional tasks.

\section{Iterative Algorithm: ILASP2i}

In the previous section, we showed that our new $I L P_{L O A S}^{\text {context }}$ task can be translated into $I L P_{L O A S}$, and therefore solved using the ILASP2 algorithm (Law et al. 2015a). However, ILASP2 may suffer from scalability issues, due to the number of examples or the size and complexity of the grounding of the hypothesis space, when combined with the background knowledge. In this paper, we address the first scalability issue by introducing a new algorithm, ILASP2i, for solving (context-dependent) learning from ordered answer sets tasks. The algorithm iteratively computes a hypothesis by incrementally constructing a subset of the examples that are relevant to the search. These are essentially counterexamples for incorrect hypotheses. The idea of the algorithm is to incrementally build, during the computation, a set of relevant examples and, at each iterative step, to learn hypotheses with respect only to this set of relevant examples instead of the full set of given examples. Although we do not directly address the second issue of large and complicated hypothesis spaces, it is worth noting that by using the notion of context-dependent examples, the size of the background knowledge (and therefore the grounding of the hypothesis space) in a particular iteration of our algorithm may be much smaller. In fact, in Section 5 we show that the background knowledge of one learning task (learning the definition of a Hamiltonian graph) can be eliminated altogether by using contexts.

\section{Definition 5}

Consider an $I L P_{L O A S}^{\text {context }}$ learning task $T=\left\langle B, S_{M},\left\langle E^{+}, E^{-}, O^{b}, O^{c}\right\rangle\right\rangle$ and a hypothesis $H \subseteq S_{M}$. A (context-dependent) example $e x$ is relevant to $H$ given $T$ if $e x \in E^{+} \cup E^{-} \cup O^{b} \cup O^{c}$ and $B \cup H$ does not cover $e x$. 
The intuition of ILASP2i (Algorithm 1) is that we start with an empty set of relevant examples and an empty hypothesis. At each step of the search we look for an example which is relevant to our current hypothesis (i.e. an example that $B \cup H$ does not cover). If no such example exists, then we return our current hypothesis as an optimal inductive solution; otherwise, we add the example to our relevant set of examples and use ILASP2 to compute a new hypothesis.

The notation $\ll$, in line 5 of algorithm 1 1 , means to add the relevant example re to the correct set in Relevant (the first set if it is a positive example etc).

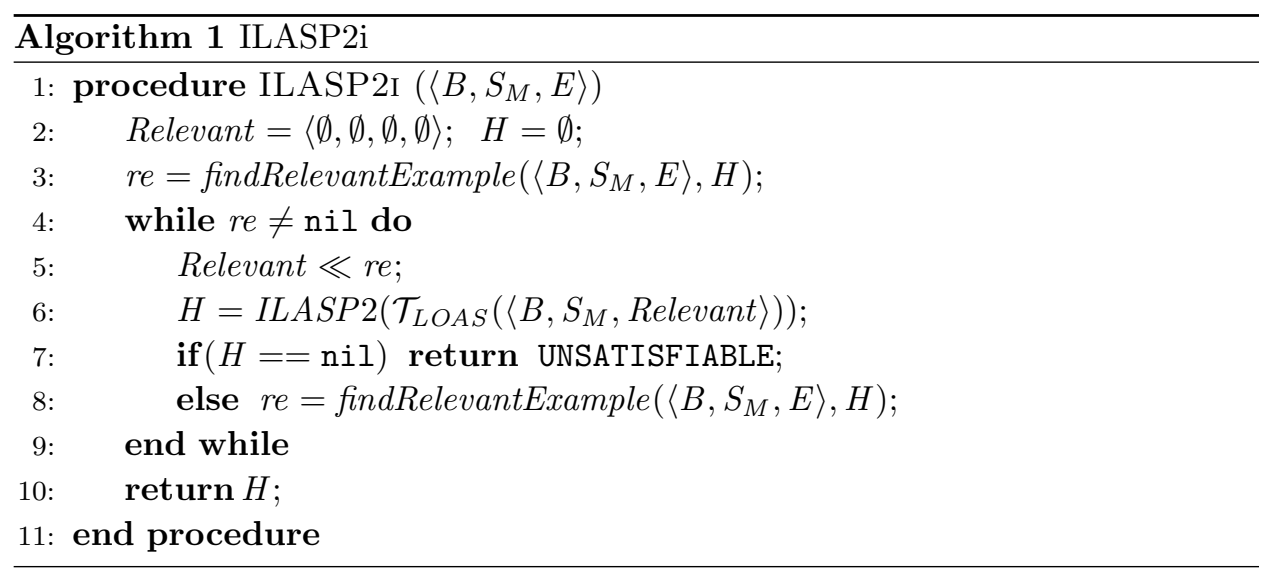

The function findRelevantExample $\left(\left\langle B, S_{M}, E\right\rangle, H\right)$ returns a (context-dependent) example in $E$ which is not covered by $B \cup H$, or nil if no such example exists. It works by encoding $B \cup H$ and $E$ into a meta program whose answer sets can be used to determine which examples in $E$ are covered. This meta program contains a choice rule, which specifies that each answer set of the program tests the coverage of a single CDPI or CDOE example. For a positive or negative example $e x=\langle e, C\rangle$, if there is an answer set of the meta program corresponding to $e x$ then there must be at least one answer set of $B \cup C \cup H$ that extends $e$. This means that positive (resp. negative) examples are covered iff there is at least one (resp. no) answer set of the meta program that corresponds to ex. Similarly, CDOE's are encoded such that each brave (resp. cautious) ordering $o$ is respected iff there is at least one (resp. no) answer set corresponding to $o$. findRelevantExamples uses the answer sets of the meta program to determine which examples are not covered. Details of the meta program are in Appendix B including proof of its correctness.

It should be noted that in the worst case our set of relevant examples is equal to the entire set of examples. In this case, ILASP2i is slower than ILASP2. In real settings, however, as examples are not carefully constructed, there is likely to be overlap between examples, so the relevant set will be much smaller than the whole set. Theorem 3 shows that ILASP2i has the same condition for termination as ILASP2.

Theorem 3

ILASP2i terminates for any well defined $I L P_{L O A S}^{\text {context }}$ task. 
Note that although the algorithm is sound, it is complete only in the sense that it always returns an optimal solution if one exists (rather than returning the full set).

\section{Theorem 4}

ILASP2i is sound for any well defined $I L P_{L O A S}^{\text {context }}$ task, and returns an optimal solution if one exists.

Note that in Algorithm 1 the translation of a context-dependent learning task is applied to the context-dependent task generated incrementally at each step of the iteration (see line 6) instead of pre-translating the full initial task. This has the advantage that the background knowledge of the translated task only contains the contexts for the relevant examples, rather than the full set. In Section 5 we compare the efficiency of ILASP2i on $I L P_{L O A S}^{c o n t e x t}$ tasks that have been pre-translated with corresponding tasks that have not been pre-translated, and demonstrate that in the latter case ILASP2i can be up to one order of magnitude faster. We refer to the application of ILASP2i with an automatic pre-translation to $I L P_{L O A S}$ as ILASP2i_pt.

\section{Evaluation}

In this section, we demonstrate the improvement in performance of ILASP2i over ILASP2, both in terms of running time and memory usage. Although there are benchmarks for ASP solvers, as ILP systems for ASP are relatively new, and solve different computational tasks, there are no benchmarks for learning ASP programs. We therefore investigate new problems. To demonstrate the increased performance of ILASP2i over ILASP2, we chose tasks with large numbers of examples. We compare the algorithms in four problem settings, each including tasks requiring different components of the $I L P_{L O A S}^{\text {context }}$ framework. We also investigate how the performance and accuracy vary with the number of examples, for the task of learning user journey preferences. All learning tasks were run with ILASP2, ILASP2i and ILASP2i_pt ${ }^{1}$.

Our first problem setting is learning the definition of whether a graph is Hamiltonian or not (i.e. whether it contains a Hamilton cycle). Hamilton A is an $I L P_{L O A S}$ (non context-dependent) task. The background knowledge $B$ consists of the two choice rules $1\{\operatorname{node}(1), \operatorname{node}(2), \operatorname{node}(3), \operatorname{node}(4)\} 4$ and $0\{\operatorname{edge}(N 1, N 2)\} 1$ :node(N1), node(N2), meaning that the answer sets of $B$ correspond to the graphs of size 1 to 4 . Each example then corresponds to exactly one graph, by specifying which node and edge atoms should be true. Positive examples correspond to Hamiltonian graphs, and negative examples correspond to non-Hamiltonian graphs. Hamilton B is an $I L P_{L O A S}^{\text {context }}$ encoding of the same problem. The background knowledge is empty, and each example has a context consisting of the node and edge atoms representing a single graph. ILASP2i performs significantly better than ILASP2 in both cases. Although ILASP2i is slightly faster at solving Hamilton B compared with Hamilton A, one interesting result is that ILASP2 and ILASP2i_pt perform better on

\footnotetext{
${ }^{1}$ For details of the tasks discussed in this section and how to download and run ILASP2, ILASP2i and ILASP2i_pt, see https://www.doc.ic.ac.uk/ ml1909/ILASP
} 


\begin{tabular}{lllllllllll}
\hline \hline \multirow{2}{*}{$\begin{array}{l}\text { Learning } \\
\text { task }\end{array}$} & \multicolumn{4}{c}{ \#examples } & \multicolumn{3}{c}{ time/s } & \multicolumn{3}{c}{ Memory/kB } \\
& $E^{+}$ & $E^{-}$ & $O^{b}$ & $O^{c}$ & 2 & $2 \mathrm{i}$-pt & $2 \mathrm{i}$ & 2 & $2 \mathrm{i}$ _pt & $2 \mathrm{i}$ \\
\hline Hamilton A & 100 & 100 & 0 & 0 & 10.3 & 4.2 & 4.3 & $9.7 \times 10^{4}$ & $1.2 \times 10^{4}$ & $1.2 \times 10^{4}$ \\
Hamilton B & 100 & 100 & 0 & 0 & 32.0 & 84.9 & 3.6 & $3.6 \times 10^{5}$ & $2.7 \times 10^{5}$ & $1.4 \times 10^{4}$ \\
Scheduling A & 400 & 0 & 110 & 90 & 291.9 & 64.2 & 63.4 & $2.7 \times 10^{6}$ & $1.7 \times 10^{5}$ & $1.7 \times 10^{5}$ \\
Scheduling B & 400 & 0 & 128 & 72 & 347.2 & 40.1 & 40.3 & $5.2 \times 10^{6}$ & $2.6 \times 10^{5}$ & $2.6 \times 10^{5}$ \\
Scheduling C & 400 & 0 & 133 & 67 & 1141.8 & 123.6 & 124.2 & $8.4 \times 10^{6}$ & $4.9 \times 10^{5}$ & $5.0 \times 10^{5}$ \\
Agent A & 200 & 0 & 0 & 0 & 444.5 & 56.7 & 39.1 & $4.7 \times 10^{6}$ & $3.7 \times 10^{5}$ & $9.8 \times 10^{4}$ \\
Agent B & 50 & 0 & 0 & 0 & TO & 212.3 & 9.4 & TO & $1.1 \times 10^{6}$ & $1.8 \times 10^{5}$ \\
Agent C & 80 & 120 & 0 & 0 & 808.7 & 132.3 & 60.1 & $2.9 \times 10^{6}$ & $3.5 \times 10^{5}$ & $8.4 \times 10^{4}$ \\
Agent D & 172 & 228 & 390 & 0 & OOM & 863.3 & 408.4 & OOM & $2.4 \times 10^{6}$ & $8.0 \times 10^{5}$ \\
\hline \hline
\end{tabular}

Table 1: The running times of ILASP2, ILASP2i and ILASP2i_pt. TO stands for time out (6 hours) and OOM stands for out of memory.

Hamilton A. This is because the non context-dependent encoding in Hamilton A is more efficient than the automatic translation (using definition 4) of Hamilton B.

To test how the size of the contexts affects the performance of the three algorithms, we reran the Hamilton A and B experiments with the maximum size of the graphs varying from 4 to 10 . Each experiment was run 100 times with randomly generated sets of positive and negative examples (100 of each in each experiment). The results (figure 1) show that ILASP2i performs best in both cases - interestingly, on average, there is no difference between Hamilton A (non context-dependent) and Hamilton B (context-dependent) at first, but as the maximum graph size increases, the domain of the background knowledge in Hamilton A increases and so ILASP2i performs better on Hamilton B. Although ILASP2i_pt is much slower on Hamilton B than Hamilton A, it uses significantly less memory on the former. As the performance of ILASP2i and ILASP2i_pt is the same on any non context-dependent task, we do not show the results for ILASP2i_pt on Hamilton A.

We also reconsider the problem of learning scheduling preferences, first presented in (Law et al. 2015a). In this setting, the goal is to learn an academic's preferences about interview scheduling, encoded as weak constraints. Tasks A-C in this case are over examples with $3 \times 3,4 \times 3$ and $5 \times 3$ timetables, respectively. As this setting contains no contexts for the examples, the performance of ILASP2i and ILASP2i_pt are relatively similar; however, for larger timetables both are over an order of magnitude faster and use over an order of magnitude less memory than ILASP2. Interestingly, although ILASP2i does not directly attempt to scale up the size of possible problem domains (in this case, the dimensions of the timetables), this experiment demonstrates that ILASP2i does (indirectly) improve the performance on larger problem domains. One unexpected observation is that ILASP2i runs faster on task B than task A. This is caused by the algorithm choosing "better" relevant examples for task $\mathrm{B}$, and therefore needing a smaller set of relevant examples. On average, the time for $4 \times 3$ timetables would be expected to be higher than the $3 \times 3$ 's. 
Iterative learning of answer set programs from context-dependent examples 11
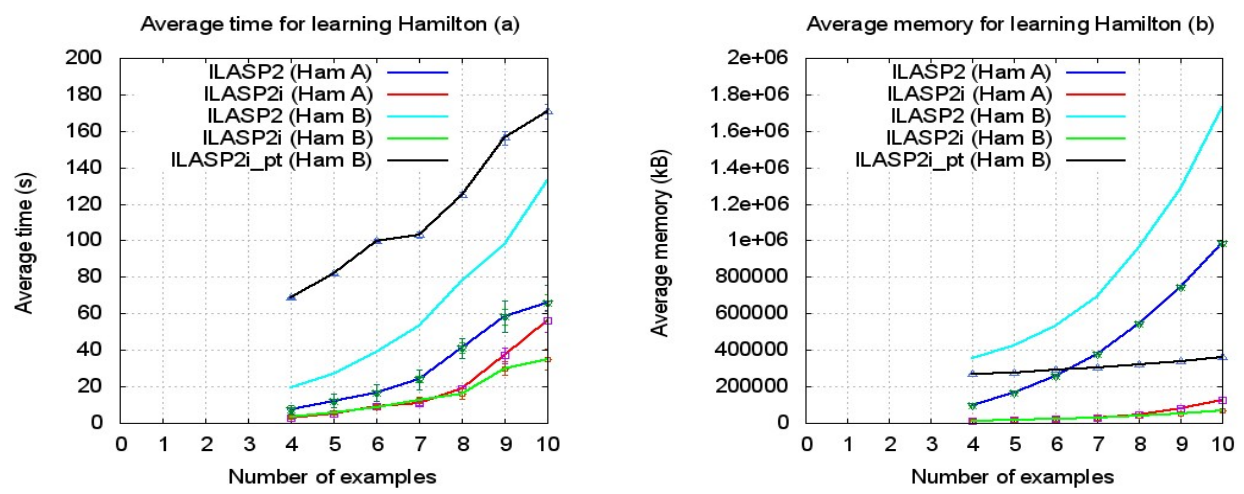

Fig. 1: (a) the average computation time and (b) the memory usage of ILASP2, ILASP2i and ILASP2i_pt for Hamilton A and B.

Our third setting is taken from (Law et al. 2014) and is based on an agent learning the rules of how it is allowed to move within a grid. Agent A requires a hypothesis describing the concept of which moves are valid, given a history of where an agent has been. Agent B requires a similar hypothesis to be learned, but with the added complexity that an additional concept is required to be invented. While Agent A and Agent B are similar to scenarios 1 and 2 in (Law et al. 2014), the key difference is that different examples contain different histories of where the agent has been. These histories are encoded as contexts, whereas in (Law et al. 2014), one single history was encoded in the background knowledge. There are also many more examples in these experiments. In Agent C, the hypothesis from Agent A must be learned along with a constraint ruling out histories in which the agent visits a cell twice (not changing the definition of valid move). This requires negative examples to be given, in addition to positive examples. In Agent D, weak constraints must be learned to explain why some traces through the grid are preferred to others. This uses positive, negative and brave ordering examples. In each case, ILASP2i performs significantly better than ILASP2i_pt, which performs significantly better than ILASP2 (ILASP2 times out in one experiment, and runs out of memory in another).

In our final setting, we investigate the problem of learning a user's preferences over alternative journeys, in order to demonstrate how the performance of the three algorithms varies with the number of examples. We also investigate how the accuracy of ILASP2i varies with the number of examples. In this scenario, a user makes requests to a journey planner to get from one location to another. The user then chooses a journey from the alternatives returned by the planner. A journey consists of one or more legs, in each of which the user uses a single mode of transport.

We used a simulation environment (Poxrucker et al. 2014) to generate realistic examples of journeys. In our experiment, we ran the simulator for one (simulated) day to generate a set of journey requests, along with the attributes of each possible journey. The attributes provided by the simulation data are: mode, which takes the value bus, car, walk or bicycle; distance, which takes an integer value between 


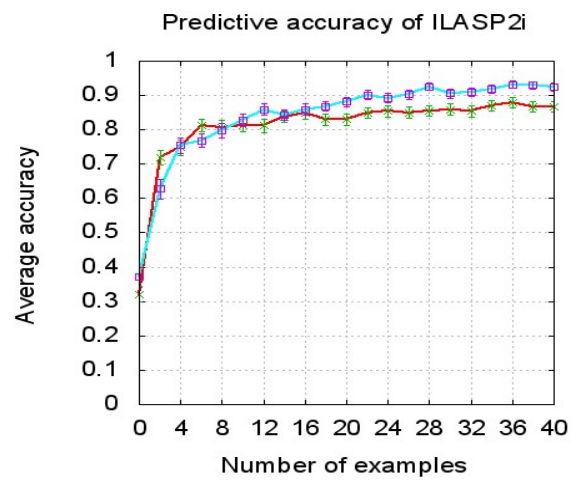

Without equality orderings With equality orderings

Fig. 2: average accuracy of ILASP2i

1 and 20000; and crime_rating. As the crime ratings were not readily available from the simulator, we used a randomly generated value between 1 and 5 .

For our experiments, we assume that the user's preferences can be represented by a set of weak constraints based on the attributes of a leg. We constructed a set of possible weak constraints, each including at most 3 literals. Most of these literals capture the leg's attributes, e.g., mode(L, bus) or crime_rating(L, R) (if the attribute's values range over integers this is represented by a variable, otherwise each possible value is used as a constant). For the crime rating (crime_rating $(L, R)$ ), we also allow comparisons of the form $R>c$ where $\mathrm{c}$ is an integer from 1 to 4 . The weight of each weak constraint is a variable representing the distance of the leg in the rule, or 1 and the priority is 1,2 or 3 . One possible set of preferences is the set of weak constraints in Example 1. $S_{J}$ denotes the set of possible weak constraints.

We now describe how to represent the journey preferences scenario in $I L P_{L O A S}^{\text {context }}$. We assume a journey is encoded as a set of attributes of the legs of the journey; for example the journey $\{$ distance $(\operatorname{leg}(1), 2000)$, distance $(\operatorname{leg}(2), 100)$, mode $(\operatorname{leg}(1)$, bus $), \operatorname{mode}(\operatorname{leg}(2)$, walk) $\}$ has two legs; in the first leg, the person must take a bus for $2000 \mathrm{~m}$ and in the second, he/she must walk $100 \mathrm{~m}$. Given a set of such journeys $J=\left\{j_{1}, \ldots, j_{n}\right\}$ and a partial ordering $O$ over $J, \mathcal{M}\left(J, O, S_{J}\right)$ is the $I L P_{L O A S}^{\text {context }}$ task $\left\langle\emptyset, S_{J}, E^{+}, \emptyset, O^{b}, \emptyset\right\rangle$, where $E^{+}=\left\{\left\langle\langle\emptyset, \emptyset\rangle, j_{i}\right\rangle \mid j_{i} \in J\right\}$ and $O^{b}=$ $\left\{\left\langle\left\langle\langle\emptyset, \emptyset\rangle, j_{1}\right\rangle,\left\langle\langle\emptyset, \emptyset\rangle, j_{2}\right\rangle\right\rangle \mid\left\langle j_{1}, j_{2}\right\rangle \in O\right\}$. Each solution of $\mathcal{M}\left(J, O, S_{J}\right)$ is a set of weak constraints representing preferences which explain the ordering of the journeys. Note that the positive examples are automatically satisfied as the (empty) background knowledge (combined with the context) already covers them. Also, as the background knowledge together with each context has exactly one answer set, the notions of brave and cautious orderings coincide; hence, we do not need cautious ordering examples for this task. Furthermore, since we are only learning weak constraints, and not hard constraints, the task also has no negative examples (a negative example would correspond to an invalid journey).

In each experiment we randomly selected 100 test hypotheses, each consisting of between 1 and 3 weak constraints from $S_{J}$. For each test hypothesis $H_{T}$, we then used the simulated journeys to generate a set of ordering examples $\left\langle j_{1}, j_{2}\right\rangle$ such that 

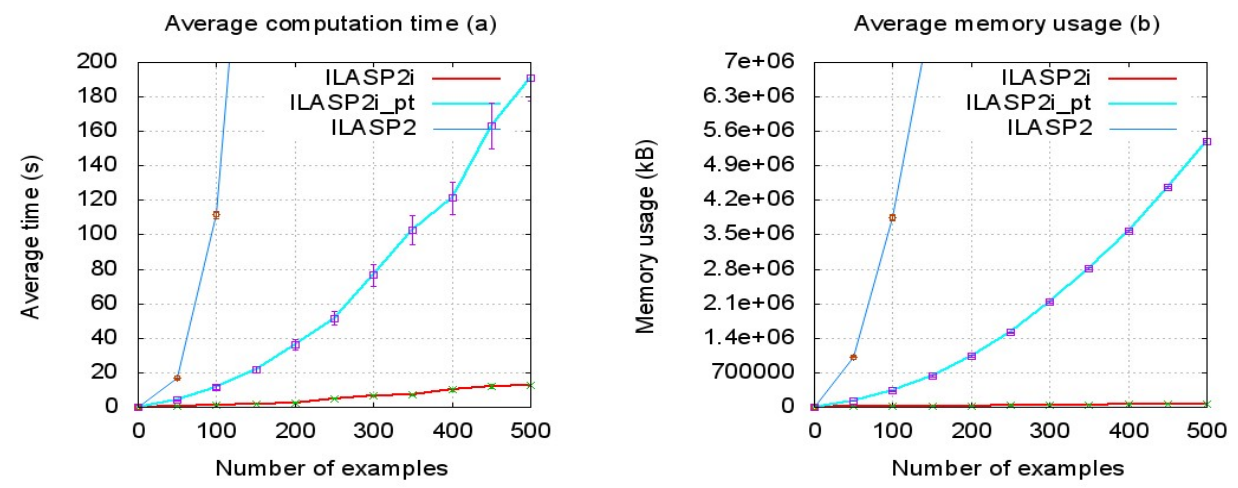

Fig. 3: (a) the average computation time and (b) the memory usage of ILASP2, ILASP2i and ILASP2i_pt for learning journey preferences.

$j_{1}$ was one of the optimal journeys, given $H$, and $j_{2}$ was an non-optimal alternative to $j_{1}$. We then tested the algorithms on tasks with varying numbers of ordering examples by taking a random sample of the complete set of ordering examples.

The accuracy of ILASP2i for different numbers of examples is shown in Figure 2. The average accuracy converges to around 85\% after roughly 20 examples. As we only gave examples of journeys such that one was preferred to the other the hypotheses were often incorrect at predicting that two journeys were equal. We therefore introduced a new type of brave ordering example to ILASP2i, which enables us to specify that two answer sets should be equally optimal. We ran the same experiment with half of the ordering examples as the new "equality" orderings. The average accuracy increased to around 93\% after 40 examples. Note that as ILASP2 and ILASP2i return an arbitrary optimal solution of a task, their accuracy results, on average, are the same. We therefore only present the results for ILASP2i.

Figures $3(\mathrm{a})$ and (b) show the running times and memory usage (respectively) for up to 500 examples for ILASP2, ILASP2i and ILASP2i_pt. For experiments with more than 200 examples, ILASP2 ran out of memory. By 200 examples, ILASP2i is already over 2 orders of magnitude faster and uses over 2 orders of magnitude less memory than ILASP2, showing a significant improvement in scalability. The fact that by 500 examples ILASP2i is an order of magnitude faster without the pre-translation shows that, in this problem domain, the context is a large factor in this improvement; however, ILASP2i_pt's significantly improved performance over ILASP2 shows that the iterative nature of ILASP2i is also a large factor.

\section{Related Work}

Most approaches to ILP address the learning of definite programs (Srinivasan 2001. Muggleton et al. 2014), usually aiming to learn Prolog programs. As the language features of Prolog and ASP are different (e.g. ASP lacks lists, Prolog lacks choice), a comparison is difficult. On the shared language of ASP and the fragment of Prolog learned by these systems (definite rules), a traditional ILP task can be represented 
with a single positive example (where the inclusions (resp. exclusions) of this example correspond to the positive (resp. negative) examples in the original task).

The idea of context-dependent example has similarities with the concept of learning from interpretation transitions (LFIT) (Inoue et al. 2014), where examples are pairs of set of atoms $\langle I, J\rangle$ such that $B \cup H$ must satisfy $T_{B \cup H}(I)=J$ (where $T_{P}(I)$ is the set of immediate consequences of $I$ with respect to the program $P$ ). LFIT technically learns under the supported model semantics and uses a far smaller language than that supported by $I L P_{L O A S}^{c o n t e x t}$ (not supporting choice rules or hard or weak constraints), but can be simply represented in $I L P_{L O A S}^{c o n t e x t}$. The head $\mathrm{h}$ of each rule in the background knowledge and hypothesis space should be replaced by $j(\mathrm{~h})$, and each body literal $\mathrm{b}$, by $\mathrm{i}(\mathrm{b})$. Each example $\langle I, J\rangle$ should then be mapped to a context-dependent positive example $\langle\langle\{j(\mathrm{a}) \mid \mathrm{a} \in J\}, \emptyset\rangle,\{\mathrm{i}(\mathrm{a}) . \mid \mathrm{a} \in I\}\rangle$.

Other than our own frameworks, the two main ILP frameworks under the answer set semantics are brave and cautious induction (Sakama and Inoue 2009). As $I L P_{L O A S}^{\text {context }}$ subsumes $I L P_{L O A S}, I L P_{L O A S}^{\text {context }}$ inherits the ability to perform both brave and cautious induction. ILASP2i is therefore more general than systems such as (Ray 2009, Corapi et al. 2012, Athakravi et al. 2014), which can only perform brave induction. In ILP, learners can be divided into batch learners (those which consider all examples simultaneously), such as (Ray 2009: Corapi et al. 2012 Athakravi et al. 2014, Law et al. 2014, and learners which consider each example in turn (using a cover loop), such as (Srinivasan 2001; Muggleton 1995, Ray et al. 2003). Under the answer set semantics, most learners are batch learners due to the non-monotonicity. In fact, it is worth noting that, in particular, although the HAIL (Ray et al. 2003) algorithm for learning definite clauses employs a cover loop, the later XHAIL algorithm is a batch learner as it learns non-monotonic programs (Ray 2009). One approach which did attempt to utilise a cover loop is (Sakama 2005). Their approach, however, was only sound for a small (monotonic) fragment of ASP if the task had multiple examples, as otherwise later examples could cause earlier examples to become uncovered.

The ILED system (Katzouris et al. 2015) extended the ideas behind XHAIL in order to allow incremental learning of event definitions. This system takes as input, multiple "windows" of examples and incrementally learns a hypothesis. As the approach is based on theory revision (at each step, revising the hypothesis from the previous step), ILED is not guaranteed to learn an optimal solution. In contrast, ILASP2i learns a new hypothesis in each iteration and incrementally builds the set of relevant examples.

\section{Conclusion}

In this paper, we have presented an extension to our $I L P_{L O A S}$ framework which allows examples to be given with extra background knowledge called the context of the example. We have shown that these contexts can be used to give structure to the background knowledge, showing which parts apply to which examples. We have also presented a new algorithm, ILASP2i, which makes use of this added structure to 
improve the efficiency over the previous ILASP2. In Section 5, we demonstrated that our new approach is considerably faster for tasks with large numbers of examples.

Unlike previous systems for learning under the answer set semantics, ILASP2i is not a batch learner and does not need to consider all examples at the same time, but instead iteratively builds a set of relevant examples. This combination of relevant examples and the added structure given by contexts means that ILASP2i can be up to 2 orders of magnitude better than ILASP2, both in terms of time and memory usage. In future work, we intend to investigate how to improve the scalability of ILASP2i with larger hypothesis spaces and with noisy examples.

\section{References}

Athakravi, D., Corapi, D., Broda, K., And Russo, A. 2014. Learning through hypothesis refinement using answer set programming. In Inductive Logic Programming. Springer, 31-46.

Corapi, D., Russo, A., And Lupu, E. 2012. Inductive logic programming in answer set programming. In Inductive Logic Programming. Springer, 91-97.

Inoue, K., Ribeiro, T., And SAKAma, C. 2014. Learning from interpretation transition. Machine Learning 94, 1, 51-79.

Katzouris, N., Artikis, A., And Paliouras, G. 2015. Incremental learning of event definitions with inductive logic programming. Machine Learning 100, 2-3, 555-585.

Law, M., Russo, A., And Broda, K. 2014. Inductive learning of answer set programs. In Logics in Artificial Intelligence (JELIA 2014). Springer.

Law, M., Russo, A., And Broda, K. 2015a. Learning weak constraints in answer set programming. Theory and Practice of Logic Programming 15, 4-5, 511-525.

Law, M., Russo, A., And Broda, K. 2015b. Proof of the soundness and completeness of ILASP2. https://www.doc.ic.ac.uk/ ml1909/Proofs_for_ILASP2.pdf

Lifschitz, V. And Turner, H. 1994. Splitting a logic program. In ICLP. Vol. 94. 23-37.

Muggleton, S. 1991. Inductive logic programming. New generation computing 8, 4, $295-318$.

Muggleton, S. 1995. Inverse entailment and progol. New generation computing 13, 3-4, $245-286$.

Muggleton, S. H., Lin, D., Pahlavi, N., And Tamaddoni-Nezhad, A. 2014. Metainterpretive learning: application to grammatical inference. Machine Learning 94, 1, 25-49.

Poxrucker, A., Bahle, G., And Lukowicz, P. 2014. Towards a real-world simulator for collaborative distributed learning in the scenario of urban mobility. In Proceedings of the Eighth IEEE International Conference on Self-Adaptive and Self-Organizing Systems Workshops. IEEE Computer Society, 44-48.

RAY, O. 2009. Nonmonotonic abductive inductive learning. Journal of Applied Logic 7, 3, 329-340.

Ray, O., Broda, K., And Russo, A. 2003. Hybrid abductive inductive learning: A generalisation of progol. In Inductive Logic Programming. Springer, 311-328.

SAKAMA, C. 2005. Induction from answer sets in nonmonotonic logic programs. ACM Transactions on Computational Logic (TOCL) 6, 2, 203-231.

Sakama, C. And Inoue, K. 2009. Brave induction: a logical framework for learning from incomplete information. Machine Learning 76, 1, 3-35.

Srinivasan, A. 2001. The aleph manual. Machine Learning at the Computing Laboratory, Oxford University. 


\section{Appendix A Proofs}

In this section, we give the proofs of the theorems in the main paper. First, we prove the preliminary lemma (Lemma 1). Really, this is a corollary of the splitting set theorem (Lifschitz and Turner 1994). $e_{U}(P, X)$ is the partial evaluation of $P$ with respect to $X$ (over the atoms in $U$ ), which is described in (Lifschitz and Turner 1994).

\section{Lemma 1}

For any program $P$ (consisting of normal rules, choice rules and constraints) and any set of pairs $S=\left\{\left\langle C_{1}, \mathrm{a}_{1}\right\rangle, \ldots,\left\langle C_{n}, \mathrm{a}_{\mathrm{n}}\right\rangle\right\}$ such that none of the atoms $\mathrm{a}_{\mathrm{i}}$ appear in $P$ (or in any of the $C$ 's) and each $\mathrm{a}_{\mathrm{i}}$ atom is unique: $A S\left(P \cup\left\{1\left\{\mathrm{a}_{1}, \ldots, \mathrm{a}_{\mathrm{n}}\right\} 1.\right\} \cup\right.$ $\left.\left\{\operatorname{append}\left(C_{i}, \mathrm{a}_{\mathrm{i}}\right) \mid\left\langle C_{i}, \mathrm{a}_{\mathrm{i}}\right\rangle \in S\right\}\right)=\left\{A \cup\left\{\mathrm{a}_{\mathrm{i}}\right\} \mid A \in A S\left(P \cup C_{i}\right),\left\langle C_{i}, \mathrm{a}_{\mathrm{i}}\right\rangle \in S\right\}$

Proof

The answer sets of $\left\{1\left\{a_{1}, \ldots, a_{n}\right\} 1.\right\}$ are $\left\{a_{1}\right\}, \ldots,\left\{a_{n}\right\}$, hence by the splitting set theorem (using $U=\left\{\mathrm{a}_{1}, \ldots, \mathrm{a}_{\mathrm{n}}\right\}$ as a splitting set):

$$
\begin{aligned}
& A S\left(P \cup\left\{1\left\{\mathrm{a}_{1}, \ldots, \mathrm{a}_{\mathrm{n}}\right\} 1 .\right\} \cup\left\{\operatorname{append}\left(C_{i}, \mathrm{a}_{\mathrm{i}}\right) \mid\left\langle C_{i}, \mathrm{a}_{\mathrm{i}}\right\rangle \in S\right\}\right)
\end{aligned}
$$

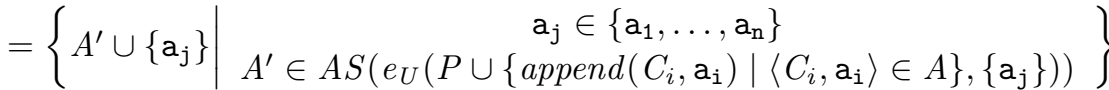

$$
\begin{aligned}
& =\left\{A \cup\left\{\mathrm{a}_{\mathrm{i}}\right\} \mid A \in A S\left(P \cup C_{i}\right),\left\langle C_{i}, \mathrm{a}_{\mathrm{i}}\right\rangle \in S\right\} \text {. }
\end{aligned}
$$

\section{Theorem 2}

The complexity of deciding whether an $I L P_{L O A S}^{\text {context }}$ task is satisfiable is $\Sigma_{2}^{P}$-complete.

\section{Proof}

Deciding satisfiability for $I L P_{L O A S}$ is $\Sigma_{2}^{P}$-complete ((Law et al. 2015a)). It is therefore sufficient to show that there is a polynomial mapping from $I L P_{L O A S}$ to $I L P_{L O A S}^{\text {context }}$ and a polynomial mapping from $I L P_{L O A S}^{\text {context }}$ to $I L P_{L O A S}$. The former is trivial (any $I L P_{L O A S}$ task can be mapped to the same task in $I L P_{L O A S}^{\text {context }}$ with empty contexts). The latter follows from theorem 1 .

Theorem 3

ILASP2i terminates for any well defined $I L P_{L O A S}^{\text {context }}$ task.

Proof

Assume that the task $T=\left\langle B, S_{M}, E\right\rangle$ is well defined. This means that $T_{1}=$ $\mathcal{T}_{L O A S}(T)$ is a well defined $I L P_{L O A S}$ task (every possible hypothesis has a finite grounding when combined with the background knowledge of $T_{1}$ ). Note that this also means that $T_{2}=\mathcal{T}_{L O A S}\left(\left\langle B, S_{M}\right.\right.$, Relevant $\left.\rangle\right)$ is well defined in each iteration as the size of the grounding of the background knowledge of $T_{2}$ combined with each hypothesis will be smaller than or equal to the size of the background in $T_{1}$ (the background knowledge of $T_{2}$ is almost a subset of the background in $T_{1}$, other than the extra choice rule, which is smaller).

The soundness of ILASP2 (Law et al. 2015a) can be used to show that $H$ will 
always cover every example in Relevant; hence, at each step re must be an example which is in $E$ but not in Relevant. As there are a finite number of examples in $E$, this means there can only be a finite number of iterations; hence, it remains to show that each iteration terminates. This is the case because, as $\mathcal{T}_{L O A S}\left(\left\langle B, S_{M}\right.\right.$, Relevant $\left.\rangle\right)$ is well defined, the call to ILASP2 terminates ( $($ Law et al. 2015a)) and findRelevantExample terminates (Appendix B.

Theorem 4

ILASP2i is sound for any well defined $I L P_{L O A S}^{\text {context }}$ task, and returns an optimal solution if one exists.

Proof

If the ILASP2i algorithm returns a hypothesis then the while loop must terminate. For this to happen findRelevantExample must return nil. This means that $H$ must cover every example in $E$. Hence ILASP2i is sound. As the algorithm terminates (see Theorem 3), the only way for a solution not to be returned is when ILASP2 returns nil. Since ILASP2 is complete (Law et al. 2015a), this is only possible when $\left\langle B, S_{M}\right.$, Relevant $\rangle$ is unsatisfiable. But if $\left\langle B, S_{M}\right.$, Relevant $\rangle$ is unsatisfiable then so is $\left\langle B, S_{M}, E\right\rangle$.

It remains to show that when a solution is returned, it is an optimal solution. Any solution $H$ returned must be an optimal solution of $\left\langle B, S_{M}\right.$, Relevant $\rangle$, (as ILASP2 returns an optimal solution). As it must also be a solution of $\left\langle B, S_{M}, E\right\rangle$, it must be an optimal solution (any shorter solution would be a solution of $\left\langle B, S_{M}\right.$, Relevant $\rangle$, contradicting that $H$ is an optimal solution for $\left\langle B, S_{M}\right.$, Relevant $\rangle$ ).

\section{Appendix B findRelevantExamples}

In this section, we describe (and prove the correctness of) the findRelevantExamples method which was omitted from the main paper. The method uses a meta encoding in ASP. Given a learning task and a hypothesis from the hypothesis space, this meta encoding is used to compute the set of examples that are covered and the set that are not covered. The meta encoding is formalised in definition 9 , but we first introduce some notation in order to simplify the main definition. Some definitions are similar to those used in the ILASP2 meta representation (Law et al. 2015a).

\section{Definition 6}

For any ASP program $P$ and predicate name pred, reify $(P$, pred) denotes the program constructed by replacing every atom a $\in P^{\prime}$ (where $P^{\prime}$ is $P$ with the weak constraints removed) by pred(a). We use the same notation for sets of literals/partial interpretations, so for a set $S: \operatorname{reify}(S$, pred $)=\{\operatorname{pred}($ atom $):$ atom $\in S\}$.

Definition 7 formalises the way we represent weak constraints in our meta encoding. We use this representation to check whether ordering examples are covered. We use as1 and as2 to represent the atoms in two answer sets (as1 and as2 occur elsewhere in our encoding). The w atoms are then used to capture the penalties paid by each answer set at each level. 


\section{Definition 7}

For any ASP program $P$, we write weak $(P)$ to mean the program constructed from the weak constraints in $P$, translating each weak constraint $: \sim \mathrm{b}_{1}, \ldots, \mathrm{b}_{\mathrm{m}}$, not $b_{m+1}, \ldots$, not $b_{n} \cdot\left[1\right.$ ev@ $\left.@_{w t}, t_{1}, \ldots, t_{k}\right]$ to the rules:

$$
\left\{\begin{aligned}
\mathrm{w}\left(\mathrm{wt}, \mathrm{lev}, \operatorname{terms}\left(\mathrm{t}_{1}, \ldots, \mathrm{t}_{\mathrm{k}}\right), \text { as } 1\right):-\operatorname{as} 1\left(\mathrm{~b}_{1}\right), \ldots, \operatorname{as} 1\left(\mathrm{~b}_{\mathrm{m}}\right), \\
\mathrm{not} \operatorname{as} 1\left(\mathrm{~b}_{\mathrm{m}+1}\right), \ldots, \operatorname{not} \operatorname{as} 1\left(\mathrm{~b}_{\mathrm{n}}\right) . \\
\mathrm{w}\left(\mathrm{wt}, \mathrm{lev}, \operatorname{terms}\left(\mathrm{t}_{1}, \ldots, \mathrm{t}_{\mathrm{k}}\right), \operatorname{as} 2\right):-\operatorname{as} 2\left(\mathrm{~b}_{1}\right), \ldots, \operatorname{as} 2\left(\mathrm{~b}_{\mathrm{m}}\right), \\
\operatorname{not} \operatorname{as} 2\left(\mathrm{~b}_{\mathrm{m}+1}\right), \ldots, \operatorname{not} \operatorname{as} 2\left(\mathrm{~b}_{\mathrm{n}}\right) .
\end{aligned}\right\}
$$

We now introduce a simplified version of the ASP program fragment which is used by ILASP2 to check whether one answer set dominates another. This is used in determining whether an ordering example is covered by a hypothesis. This makes use of the w atoms which are generated by the w rules in definition 7 and captures the definition of dominates given in Section 2 .

\section{Definition 8}

dominates is the program:

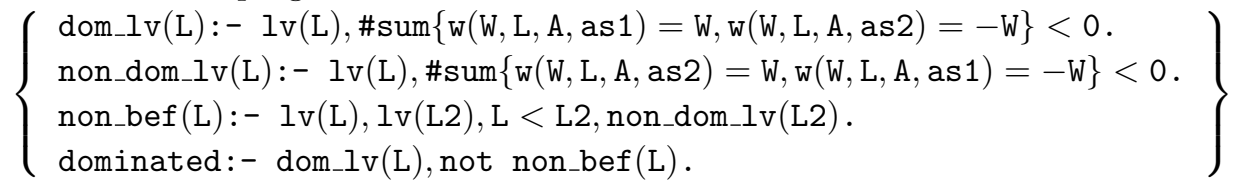

In (Law et al. 2015a), multiple instances of dominates were included in the same meta encoding, and hence the program was slightly more complicated in order to track the different instances. The main structure of the program is the same however, and hence the same results apply. The result we need for this paper is proven (for the more general program) in (Law et al. 2015b) and is given by Lemma 2 .

\section{Lemma 2}

Let $I_{1}$ and $I_{2}$ be interpretations, $P$ be an ASP program and $L$ be the set of levels used in the weak constraints in $P$. The unique answer set of dominates $\cup\{\operatorname{lv}(1)$. | $l \in L\} \cup$ weak $(P) \cup \operatorname{reify}\left(I_{1}\right.$, as 1$) \cup$ reify $\left(I_{1}\right.$, as2 $)$ contains the atom dominated if and only if $I_{1}$ dominates $I_{2}$ wrt the weak constraints in $P$.

Definition 9 captures the meta encoding we use in findRelevantExamples. This encoding is made of 6 components. $\mathcal{R}_{1}$ captures the background knowledge and hypothesis - by reifying $B \cup H$, the as1 and as2 atoms represent two answer sets $A_{1}$ and $A_{2}$, and the dominates program (together with weak $(B \cup H)$ and the priority levels) checks whether $A_{1}$ dominates $A_{2}$. The programs $\mathcal{R}_{2}$ to $\mathcal{R}_{5}$ are used to check whether each type of example is covered. These programs make use of the predicate test_on of arity 2 and the test predicate of arity 1 . The meaning of test $\left(e_{i d}\right)$ is that the example ex should be tested. There is a choice rule in $\mathcal{R}_{6}$ to say that each example should be tested. For the positive and negative examples, this means that they should be tested on as1 (meaning to check whether it is possible that an answer set of $B \cup H$ extends this example). For an ordering example $\left\langle e x_{1}, e x_{2}\right\rangle$ it is slightly more involved: $e x_{1}$ should be tested on as 1 and $e x_{2}$ should be tested on as2 (and the ordering should be checked). 
Iterative learning of answer set programs from context-dependent examples 19

Definition 9

Let $T$ be the $I L P_{L O A S}^{\text {context }}$ task $\left\langle B, S_{M},\left\langle E^{+}, E^{-}, O^{b}, O^{c}\right\rangle\right\rangle$ and $H$ be a hypothesis such that $H \subseteq S_{M}$. Let $L$ be the set of all priority levels in $B \cup H \mathcal{R}(T, H)$ is the ASP program $\mathcal{R}_{1}(B \cup H) \cup \mathcal{R}_{2}\left(E^{+}\right) \cup \mathcal{R}_{3}\left(E^{-}\right) \cup \mathcal{R}_{4}\left(O^{b}\right) \cup \mathcal{R}_{5}\left(O^{c}\right) \cup \mathcal{R}_{6}\left(E^{+} \cup E^{-}, O^{b} \cup O^{c}\right)$, where the individual components are as follows:

- $\mathcal{R}_{1}(B \cup H)=\operatorname{reify}(B \cup H$, as1 $) \cup \operatorname{reify}(B \cup H$, as2 $) \cup$ weak $(B \cup H) \cup\{\operatorname{lv}(1)$.

$l \in L\} \cup$ dominates

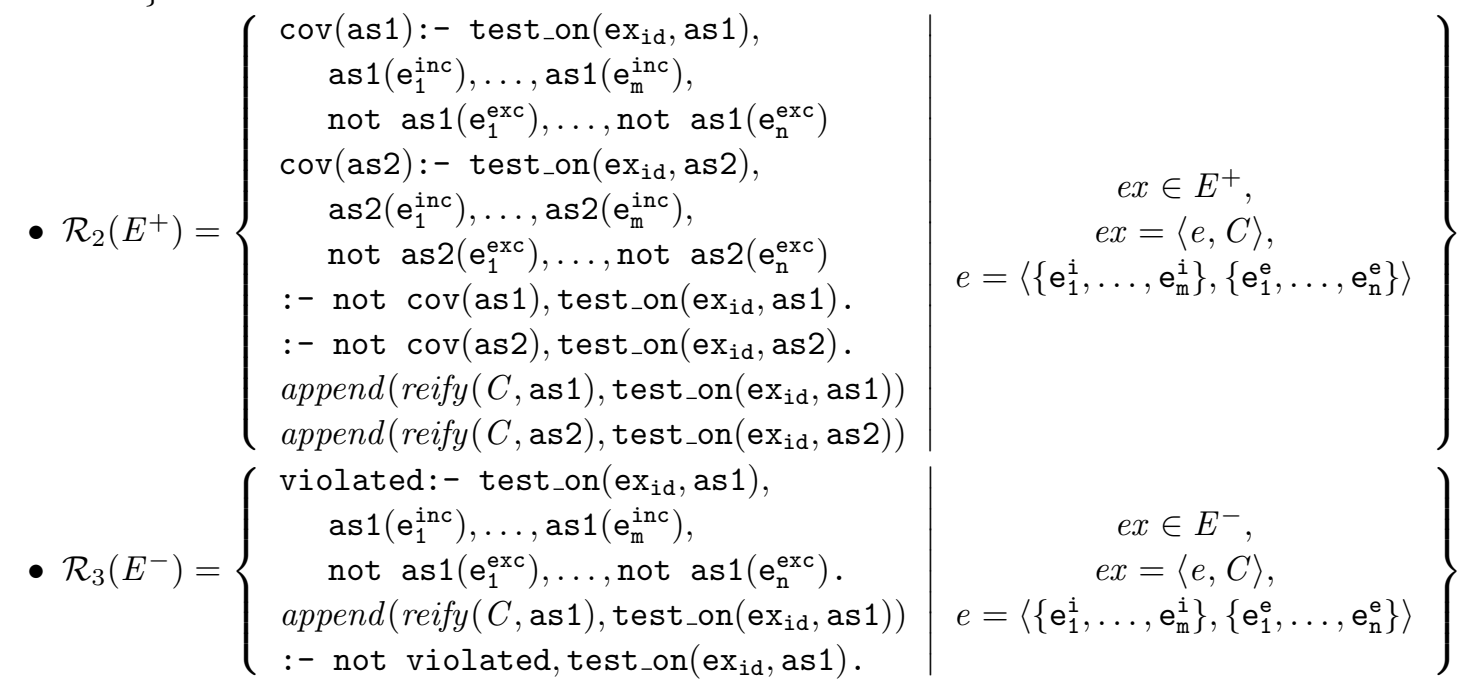

$$
\begin{aligned}
& \text { - } \mathcal{R}_{4}\left(O^{b}\right)=\left\{:-\operatorname{test}\left(\mathrm{o}_{\mathrm{id}}\right), \text { not dominated. } \mid o \in O^{b}\right\} \\
& \text { - } \mathcal{R}_{5}\left(O^{c}\right)=\left\{\text { :- test }\left(\mathrm{o}_{\text {id }}\right) \text {, dominated. } \mid o \in O^{c}\right\} \\
& \text { - } \mathcal{R}_{6}\left(\left\{e x_{1}, \ldots e x_{m}\right\},\left\{o_{1}, \ldots o_{n}\right\}\right)=\left\{1\left\{\operatorname{test}\left(\operatorname{ex}_{1}\right), \ldots, \operatorname{test}\left(\operatorname{ex}_{\mathrm{m}}\right), \operatorname{test}\left(o_{1}\right), \ldots, \operatorname{test}\left(o_{\mathrm{n}}\right)\right\} 1 .\right\} \\
& \cup\left\{\text { test_on }\left(e x_{i}, \text { as } 1\right):-\operatorname{test}\left(e_{i}\right) . \mid e x_{i} \in\left\{e x_{1}, \ldots, e x_{m}\right\}\right\} \\
& \cup\left\{\begin{array}{c|c}
\text { test_on }\left(\operatorname{ex}_{1}, \text { as } 1\right):-\operatorname{test}\left(\mathrm{o}_{\mathrm{i}}\right) . & o_{i} \in\left\{o_{1}, \ldots, o_{n}\right\} \\
\text { test_on }\left(\operatorname{ex}_{2}, \text { as2 }\right):-\operatorname{test}\left(\mathrm{o}_{\mathrm{i}}\right) . & o_{i}=\left\langle e x_{1}, e x_{2}\right\rangle
\end{array}\right\}
\end{aligned}
$$

Theorem 5

Let $T$ be any $I L P_{L O A S}^{c o n t e x t}$ task and $H$ be any subset of the hypothesis space.

1. $\forall e x \in E^{+}, \exists A \in A S(\mathcal{R}(T, H))$ st test $\left(\operatorname{ex}_{\text {id }}\right) \in A$ iff $H$ covers $e x$.

2. $\forall e x \in E^{-}, \exists A \in A S(\mathcal{R}(T, H))$ st test $\left(\operatorname{ex}_{\text {id }}\right) \in A$ iff $H$ does not cover $e x$.

3. $\forall o \in O^{b}, \exists A \in A S(\mathcal{R}(T, H))$ st test $\left(\mathrm{o}_{\mathrm{id}}\right) \in A$ iff $H$ bravely respects $o$.

4. $\forall o \in O^{c}, \exists A \in A S(\mathcal{R}(T, H))$ st test $\left(\mathrm{o}_{\mathrm{id}}\right) \in A$ iff $H$ does not cautiously respect $o$.

Proof

1. Let $e x=\langle e, C\rangle$ be a CDPI in $E^{+}$st $e=\left\langle\left\{\mathrm{e}_{1}^{\mathrm{i}}, \ldots \mathrm{e}_{\mathrm{m}}^{\mathrm{i}}\right\},\left\{\mathrm{e}_{1}^{\mathrm{e}}, \ldots, \mathrm{e}_{\mathrm{n}}^{\mathrm{e}}\right\}\right\rangle$.

$H$ covers $e x \Leftrightarrow \exists A \in A S(B \cup H \cup C)$ st $A$ extends $e$ $\Leftrightarrow \exists A \in A S(\operatorname{reify}(B \cup H \cup C$, as1) $)$ st $A$ extends reify(e, as1)

$$
\Leftrightarrow \operatorname{reify}(B \cup H \cup C, \operatorname{as} 1) \cup\left\{\begin{array}{c}
\operatorname{cov}(\operatorname{as} 1):-\operatorname{as} 1\left(\mathrm{e}_{1}\right), \ldots, \operatorname{as} 1\left(\mathrm{e}_{\mathrm{m}}\right), \\
\operatorname{not} \operatorname{as} 1\left(\mathrm{e}_{1}\right), \ldots, \operatorname{not} \operatorname{as} 1\left(\mathrm{e}_{\mathrm{n}}\right) . \\
:-\operatorname{not} \operatorname{cov}(\operatorname{as} 1) .
\end{array}\right\} \text { is }
$$

satisfiable (we refer to this program as $P_{1}$ later in the proof). 
$\begin{aligned} & \Leftrightarrow \operatorname{reify}(B \cup H, \operatorname{as} 1) \cup \operatorname{append}\left(\operatorname{reify}(C, \text { as1 }), \text { test_on }\left(\mathrm{ex}_{\mathrm{id}}, \text { as } 1\right)\right) \\ & \cup\left\{\begin{array}{c}\operatorname{cov}(\operatorname{as} 1):- \text { test_on }\left(\mathrm{ex}_{\mathrm{id}}, \text { as } 1\right), \\ \operatorname{as} 1\left(\mathrm{e}_{1}\right), \ldots, \text { as } 1\left(\mathrm{e}_{\mathrm{m}}\right), \\ \operatorname{not} \text { as } 1\left(\mathrm{e}_{1}\right), \ldots, \text { not as } 1\left(\mathrm{e}_{\mathrm{n}}\right) . \\ :-\operatorname{not} \operatorname{cov}(\operatorname{as} 1), \text { test_on }\left(\mathrm{ex}_{\mathrm{id}}, \text { as } 1\right) .\end{array}\right\} \cup \mathcal{R}_{6}\left(E^{+} \cup E^{-}, O^{b} \cup O^{c}\right)\end{aligned}$ has an answer set which contains test $\left(\mathrm{ex}_{\mathrm{id}}\right)$ (we refer to this program as $P_{2}$ ). This follows from the splitting set theorem, using the atoms in $\mathcal{R}_{6}\left(E^{+} \cup E^{-}, O^{b} \cup O^{c}\right)$ as a splitting set $-\left\{\right.$ test $\left(\mathrm{ex}_{\mathrm{id}}\right)$, test_on $\left.\left(\mathrm{ex}_{\mathrm{id}}, \mathrm{as}_{1}\right)\right\}$ is an answer set of the bottom program, leading to $P_{1}$ as the partially evaluated top program

$\Leftrightarrow \mathcal{R}(T, H)$ has an answer set which contains test $\left(\mathrm{ex}_{\mathrm{id}}\right)$. Again, this is by the splitting set theorem, using the atoms in $\mathcal{R}_{6}\left(E^{+} \cup E^{-}, O^{b} \cup O^{c}\right)$ as a splitting set, as $P_{2} \subseteq \mathcal{R}(T, H)$ and each of the extra rules in $\mathcal{R}(T, H)$ which are not in $P_{2}$ contain a test_on or test atom in the body that is not in the answer set $\left\{\right.$ test $\left(\mathrm{ex}_{\mathrm{id}}\right)$, test_on $\left.\left(\mathrm{ex}_{\mathrm{id}}, \mathrm{as}_{1}\right)\right\}$ and hence they are removed from the partially evaluated top program.

2. Let $e x=\langle e, C\rangle$ be a CDPI in $E^{-}$st $e=\left\langle\left\{\mathrm{e}_{1}^{\mathrm{i}}, \ldots \mathrm{e}_{\mathrm{m}}^{\mathrm{i}}\right\},\left\{\mathrm{e}_{1}^{\mathrm{e}}, \ldots, \mathrm{e}_{\mathrm{n}}^{\mathrm{e}}\right\}\right\rangle$.

$H$ does not cover $e x \Leftrightarrow \exists A \in A S(B \cup H \cup C)$ st $A$ extends $e$

$\Leftrightarrow \exists A \in A S($ reify $(B \cup H \cup C$, as1 $))$ st $A$ extends reify $(e$, as1 $)$

$\Leftrightarrow \operatorname{reify}(B \cup H \cup C$, as 1$) \cup\left\{\begin{array}{c}\text { violated: }-\operatorname{as} 1\left(\mathrm{e}_{1}\right), \ldots, \operatorname{as} 1\left(\mathrm{e}_{\mathrm{m}}\right), \\ \text { not as1 }\left(\mathrm{e}_{1}\right), \ldots, \text { not } \operatorname{as} 1\left(\mathrm{e}_{\mathrm{n}}\right) . \\ :-\operatorname{not} \text { violated. }\end{array}\right\}$ is satisfiable

(we refer to this program as $P_{3}$ later in the proof)

$\Leftrightarrow \operatorname{reify}(B \cup H$, as1 $) \cup \operatorname{append}\left(\operatorname{reify}\left(C\right.\right.$, as1), test_on(ex $\mathrm{id}_{\mathrm{id}}$, as 1$\left.)\right)$

$\cup\left\{\begin{array}{c}\text { violated:- test_on }\left(\mathrm{ex}_{\mathrm{id}}, \text { as1 }\right), \\ \text { as1 }\left(\mathrm{e}_{1}\right), \ldots, \text { as } 1\left(\mathrm{e}_{\mathrm{m}}\right), \\ \text { not as } 1\left(\mathrm{e}_{1}\right), \ldots, \text { not as } 1\left(\mathrm{e}_{\mathrm{n}}\right) . \\ :- \text { not violated, test_on }\left(\mathrm{ex}_{\mathrm{id}}, \operatorname{as} 1\right) .\end{array}\right\} \cup \mathcal{R}_{6}\left(E^{+} \cup E^{-}, O^{b} \cup O^{c}\right)$

has an answer set which contains test (ex $\mathrm{id}_{\mathrm{id}}$ ) (we refer to this program as $P_{4}$ ).

This follows by the splitting set theorem, using the atoms in $\mathcal{R}_{6}\left(E^{+} \cup E^{-}, O^{b} \cup\right.$

$O^{c}$ ) as a splitting set, $\left\{\right.$ test $\left(e_{i d}\right)$, test_on $\left(e_{i d}\right.$, as 1$\left.)\right\}$ is an answer set of the bottom program, leading to $P_{3}$ as the partially evaluated top program.

$\Leftrightarrow \mathcal{R}(T, H)$ has an answer set which contains test $\left(\mathrm{ex}_{\mathrm{id}}\right)$. Again, this is by the splitting set theorem, using the atoms in $\mathcal{R}_{6}\left(E^{+} \cup E^{-}, O^{b} \cup O^{c}\right)$ as a splitting set, as $P_{4} \subseteq \mathcal{R}(T, H)$ and each of the extra rules in $\mathcal{R}(T, H)$ which are not in $P_{4}$ contain a test_on or test atom in the body that is not in the answer set $\left\{\right.$ test $\left(\mathrm{ex}_{\mathrm{id}}\right)$, test_on $\left(\mathrm{ex}_{\mathrm{id}}\right.$, as 1$\left.)\right\}$ and hence they are removed from the partially evaluated top program.

3. Let $o=\langle e x 1, e x 2\rangle$ be a CDOE in $O^{b}$ st $e x 1=\left\langle e 1, C_{1}\right\rangle$, ex $2=\left\langle e 2, C_{2}\right\rangle, e_{1}=$ $\left\langle\left\{\mathrm{e} 1_{1}^{\mathrm{i}}, \ldots, \mathrm{e} 1_{\mathrm{m}}^{\mathrm{i}}\right\},\left\{\mathrm{e} 1_{1}^{\mathrm{e}}, \ldots, \mathrm{e} 1_{\mathrm{n}}^{\mathrm{e}}\right\}\right\rangle$ and $e_{2}=\left\langle\left\{\mathrm{e} 2_{1}^{\mathrm{i}}, \ldots, \mathrm{e} 2_{\mathrm{j}}^{\mathrm{i}}\right\},\left\{\mathrm{e} 2_{1}^{\mathrm{e}}, \ldots, \mathrm{e} 2_{\mathrm{k}}^{\mathrm{e}}\right\}\right\rangle$.

$H$ bravely respects $o \Leftrightarrow \exists A_{1} \in A S\left(B \cup H \cup C_{1}\right), \exists A_{2} \in A S\left(B \cup H \cup C_{2}\right)$ st $A_{1}$ extends $e_{1}, A_{2}$ extends $e_{2}$ and $A_{1} \prec_{B \cup H} A_{2}$

$\Leftrightarrow \exists A_{1} \in A S\left(\right.$ reify $\left(B \cup H \cup C_{1}\right.$, as 1$\left.)\right), \exists A_{2} \in A S\left(\operatorname{reify}\left(B \cup H \cup C_{2}\right.\right.$, as2) $)$ st $A_{1}$ extends reify (e $e_{1}$ as 1$), A_{2}$ extends reify $\left(e_{2}\right.$, as 2$)$ and dominated is in the unique answer set of $A_{1} \cup A_{2} \cup$ weak $(B \cup H) \cup\{\mathrm{Iv}(\mathrm{I}) . \mid 1 \in L\} \cup$ dominates (by Lemma2) $\Leftrightarrow \operatorname{reify}\left(B \cup H \cup C_{1}\right.$, as 1$) \cup \operatorname{reify}\left(B \cup H \cup C_{2}\right.$, as2 $) \cup$ weak $(B \cup H) \cup\{\operatorname{lv}(1)$. | 
$1 \in L\} \cup$ dominates $)$

$$
\cup\left\{\begin{array}{c}
\operatorname{cov}(\operatorname{as} 1):-\operatorname{as} 1\left(e 1_{1}^{i}\right), \ldots, \operatorname{as} 1\left(e 1_{m}^{i}\right), \\
\operatorname{not} \operatorname{as} 1\left(e 1_{1}^{\mathrm{e}}\right), \ldots, \operatorname{not} \operatorname{as} 1\left(e 1_{n}^{\mathrm{e}}\right) . \\
:-\operatorname{not} \operatorname{cov}(\operatorname{as} 1) . \\
\operatorname{cov}(\operatorname{as} 2):-\operatorname{as} 2\left(e 2_{1}^{i}\right), \ldots, \operatorname{as} 2\left(e 2_{j}^{i}\right), \\
\quad \operatorname{not} \operatorname{as} 2\left(e 2_{1}^{\mathrm{e}}\right), \ldots, \operatorname{not} \operatorname{as} 2\left(e 2_{k}^{\mathrm{e}}\right) . \\
:-\operatorname{not} \operatorname{cov}(\operatorname{as} 2) . \\
:-\operatorname{not} d o m i n a t e d .
\end{array}\right\} \text { is satisfiable (we re- }
$$

fer to this program as $P_{5}$ later in the proof)

$\Leftrightarrow \operatorname{reify}\left(B \cup H \cup C_{1}\right.$, as 1$) \cup \operatorname{reify}\left(B \cup H \cup C_{2}\right.$, as 2$) \cup$ weak $(B \cup H) \cup\{\operatorname{lv}(1)$. | $1 \in L\} \cup$ dominates $)$

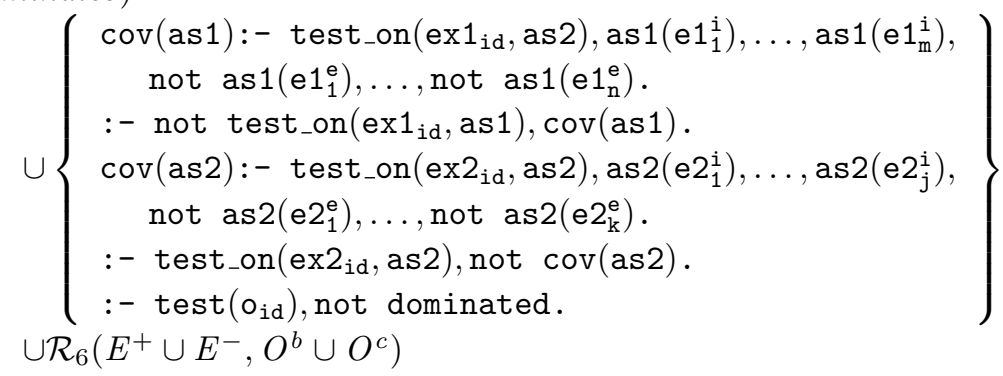

has an answer set which contains test $\left(o_{i d}\right)$ (we refer to this program as $P_{6}$ ). This follows by the splitting set theorem, using the atoms in $\mathcal{R}_{6}\left(E^{+} \cup E^{-}, O^{b} \cup O^{c}\right)$ as a splitting set, $\left\{\right.$ test $\left(o_{i d}\right)$, test_on $\left(\operatorname{ex} 1_{i d}\right.$, as 1$)$, test_on $\left.\left(e x 2_{i d}, a s 2\right)\right\}$ is an answer set of the bottom program, leading to $P_{5}$ as the partially evaluated top program

$\Leftrightarrow \mathcal{R}(T, H)$ has an answer set which contains test $\left(o_{i d}\right)$. Again, this is by the splitting set theorem, using the atoms in $\mathcal{R}_{6}\left(E^{+} \cup E^{-}, O^{b} \cup O^{c}\right)$ as a splitting set, as $P_{6} \subseteq \mathcal{R}_{6}(T, H)$ and each of the extra rules which are in $\mathcal{R}_{6}(T, H)$ but not in $P_{6}$ contain a test_on or test atom which is not in the answer set $\left\{\right.$ test $\left(o_{i d}\right)$, test_on $\left(\operatorname{ex} 1_{i d}\right.$, as 1$)$, test_on $\left.\left(\operatorname{ex} 2_{i d}, a s 2\right)\right\}$ and hence they are removed from the partially evaluated top program

4. Let $o=\langle e x 1, e x 2\rangle$ be a CDOE in $O^{c}$ st $e x 1=\left\langle e 1, C_{1}\right\rangle, e x 2=\left\langle e 2, C_{2}\right\rangle, e_{1}=$ $\left\langle\left\{\mathrm{e} 1_{1}^{\mathrm{i}}, \ldots, \mathrm{e} 1_{\mathrm{m}}^{\mathrm{i}}\right\},\left\{\mathrm{e} 1_{1}^{\mathrm{e}}, \ldots, \mathrm{e} 1_{\mathrm{n}}^{\mathrm{e}}\right\}\right\rangle$ and $e_{2}=\left\langle\left\{\mathrm{e} 2_{1}^{\mathrm{i}}, \ldots, \mathrm{e} 2_{\mathrm{j}}^{\mathrm{i}}\right\},\left\{\mathrm{e} 2_{1}^{\mathrm{e}}, \ldots, \mathrm{e} 2_{\mathrm{k}}^{\mathrm{e}}\right\}\right\rangle$

$H$ does not cautiously respect $o \Leftrightarrow \exists A_{1} \in A S\left(B \cup H \cup C_{1}\right), \exists A_{2} \in A S\left(B \cup H \cup C_{2}\right)$ st $A_{1}$ extends $e_{1}, A_{2}$ extends $e_{2}$ and $A_{1} \nprec_{B \cup H} A_{2}$

$\Leftrightarrow \exists A_{1} \in A S\left(\operatorname{reify}\left(B \cup H \cup C_{1}\right.\right.$, as 1$\left.)\right), \exists A_{2} \in A S\left(\right.$ reify $\left(B \cup H \cup C_{2}\right.$, as2)) st $A_{1}$ extends reify (e $e_{1}$,as1), $A_{2}$ extends reify $\left(e_{2}\right.$, as 2$)$ and dominated is not in the unique answer set of $A_{1} \cup A_{2} \cup$ weak $(B \cup H) \cup\{1 \mathrm{v}(1) . \mid 1 \in L\} \cup$ dominates (by Lemma 2)

$\Leftrightarrow \operatorname{reify}\left(B \cup H \cup C_{1}\right.$, as 1$) \cup \operatorname{reify}\left(B \cup H \cup C_{2}\right.$, as 2$) \cup$ weak $(B \cup H) \cup\{1 \mathrm{v}(1)$. $1 \in L\} \cup$ dominates $)$ 


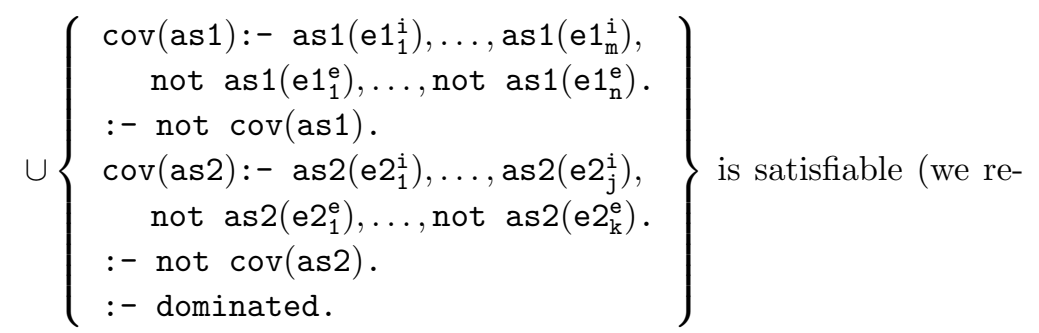

fer to this program as $P_{7}$ later in the proof)

$\Leftrightarrow \operatorname{reify}\left(B \cup H \cup C_{1}\right.$, as 1$) \cup \operatorname{reify}\left(B \cup H \cup C_{2}\right.$, as 2$) \cup$ weak $(B \cup H) \cup\{\operatorname{lv}(1)$. | $1 \in L\} \cup$ dominates $)$

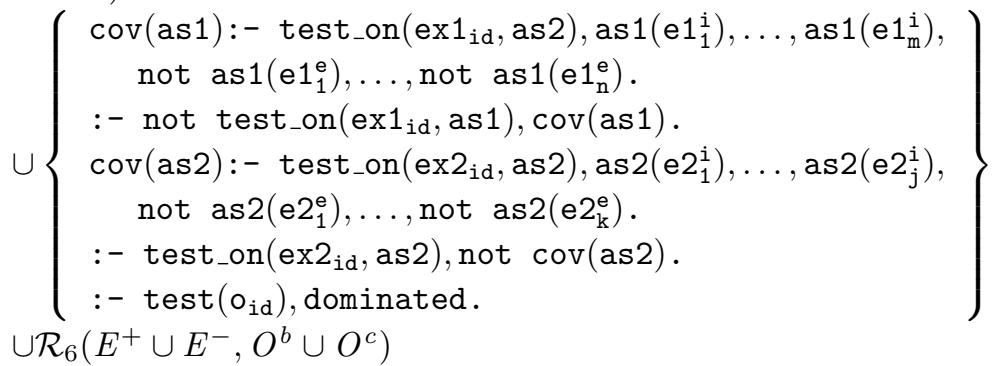

has an answer set which contains the atom test $\left(o_{i d}\right)$ (we refer to this program as $\left.P_{8}\right)$. This follows from the splitting set theorem, using the atoms in $\mathcal{R}_{6}\left(E^{+} \cup\right.$

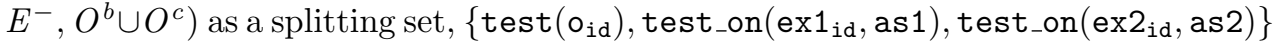
is an answer set of the bottom program, leading to $P_{7}$ as the partially evaluated top program $\Leftrightarrow \mathcal{R}(T, H)$ has an answer set which contains test $\left(o_{i d}\right)$. Again, this is by the splitting set theorem, using the atoms in $\mathcal{R}_{6}\left(E^{+} \cup E^{-}, O^{b} \cup O^{c}\right)$ as a splitting set, as $P_{8} \subseteq \mathcal{R}_{6}(T, H)$ and each of the extra rules which are in $\mathcal{R}_{6}(T, H)$ but not in $P_{8}$ contain a test_on or test atom which is not in the answer set $\left\{\operatorname{test}\left(o_{i d}\right)\right.$, test_on $\left(\operatorname{ex} 1_{i d}\right.$, as 1$)$, test_on $\left.\left(\operatorname{ex} 2_{i d}, a s 2\right)\right\}$ and hence they are removed from the partially evaluated top program.

findRelevantExamples $(T, H)$ works by constructing $\mathcal{R}(T, H)$ and computing its answer sets. For each example $e x$, whether of not $e x$ is covered by $T$ can be computed from the answer sets, using the results in Theorem 5 . The first example which is not covered is returned. If no such example is found, nil is returned. The correctness of findRelevantExamples follows directly from Theorem 5 . If the task $T$ is well defined then $\mathcal{R}(T, H)$ will ground finitely (and have a finite number of answer sets), and therefore solving $\mathcal{R}(T, H)$ for answer sets will terminate in a finite time; hence as there are a finite number of examples, findRelevantExamples will terminate in a finite time. 